DOI: $10.1002 /(($ please add manuscript number $))$

Article type: Communication

\title{
Reversible and Rewritable Surface Functionalization and Patterning via Photodynamic Disulfide Exchange
}

Xin Du, Linxian Li, Junsheng Li, Alexander Welle, Wenqian Feng, and Pavel A. Levkin*

Dr. X. Du, Dr. L. X. Li, Dr. J. S. Li, Wenqian Feng, and Dr. P. A. Levkin

Institute of Toxicology and Genetics, Karlsruhe Institute of Technology, 76021 Karlsruhe, Germany

Dr. X. Du, Dr. J. S. Li, and Dr. P.A. Levkin

Department of Applied Physical Chemistry, University of Heidelberg, 69120 Heidelberg, Germany

Dr. L. X. Li, and Wenqian Feng

Institute of Organic Chemistry, University of Heidelberg, 69120 Heidelberg, Germany Dr. A. Welle

Institute of Functional Interfaces, Karlsruhe Institute of Technology, 76021 Karlsruhe, Germany

Dr. A. Welle

Karlsruhe Nano Micro Facility, Karlsruhe Institute of Technology, 76021 Karlsruhe, Germany

Dr. P. A. Levkin

Institute of Organic Chemistry, Karlsruhe Institute of Technology, 76021 Karlsruhe, Germany

* E-mail: levkin@kit.edu

Keywords: patterning; surface functionalization; photochemistry; disulfide exchange; rewritable surface

Light-promoted precise spatial control of target molecules on surfaces is crucial in the development of novel bioanalytical, diagnostic, or sensor tools. Proteins, DNA fragments, peptides and antibodies, ${ }^{[1-6]}$ as well as hydrogels,${ }^{[7]}$ have been immobilized and patterned using a number of photochemical methods, such as thiol-yne, ${ }^{[8]}$ thiol-ene, ${ }^{[9]}$ azide-yne (by photoreduction of copper II), ${ }^{[10]}$ terazole-ene, ${ }^{[11]}$ photo-triggered Diels-Alder reaction, ${ }^{[12]}$ Paterno-Buchi reaction, ${ }^{[13]}$ and some other chemistries capable of photo-triggered formation of reactive functional groups. ${ }^{[14-17]}$ However, most of the existing photochemical methods lead to irreversible permanent surface functionalization, limiting possible applications in the formation of materials and surfaces with dynamic and responsive properties or reusable 
functionalities. Reversible surface functionalization methods can be applied to introduce, exchange, or remove a functionality and, thus, generate "smart" surfaces and patterns. Examples of possible applications of such dynamic surfaces are reusability of substrates, possibility to perform "write and erase" procedures (i.e., rewritable surfaces), formation of complex, multi-component and gradient patterns, capture-and-release properties, and the possibility of in-situ manipulation of local environments.

To the best of our knowledge, only two photo-induced reversible patterning strategies have been reported so far. Popik et al. ${ }^{[18]}$ showed that reactive $o$-naphthoquinone methides (oNQMs) produced under UV light from 3-(hydroxymethyl)-2-naphtholcould react with surface thiol groups to yield thioether conjugates, which could be subsequently cleaved by a secondary UV irradiation to regenerate surface thiol groups. ${ }^{[18]}$ In a recent publication, Anseth et al. described the use of allyl sulfides incorporated into a hydrogel to achieve reversible modification with thiol-containing biomolecules. ${ }^{[19]}$

Here, we present a new reversible photo-patterning strategy based on a photo-induced disulfide exchange reaction that allows for reversible photo-functionalization, patterning, as well as exchange or removal of surface functional groups (Figure 1).

Disulfide bonds are known to undergo reversible cleavage under basic conditions via thiol-disulfide exchange reactions through intermediate thiolate anions. ${ }^{[20-22]}$ However, disulfides can also undergo dynamic exchange reactions by homolytic photo-cleavage to become sulfanyl radicals (Figure1a). This reaction was recently adopted for the synthesis of self-healing polymers. ${ }^{[23,24]}$ We hypothesized that the dynamic nature of disulfide homolysis and recombination under UV irradiation could be utilized to achieve reversible dynamic functionalization of disulfide surfaces (Figure1b). 
(a)

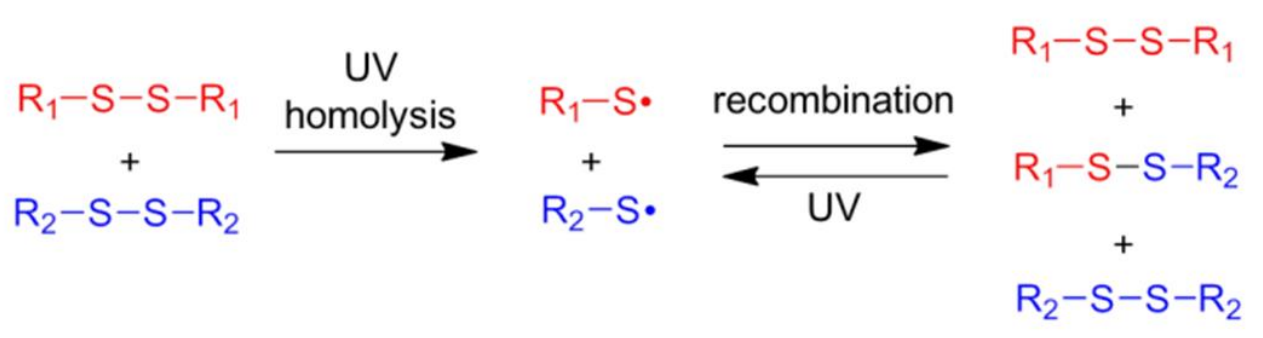

(b)

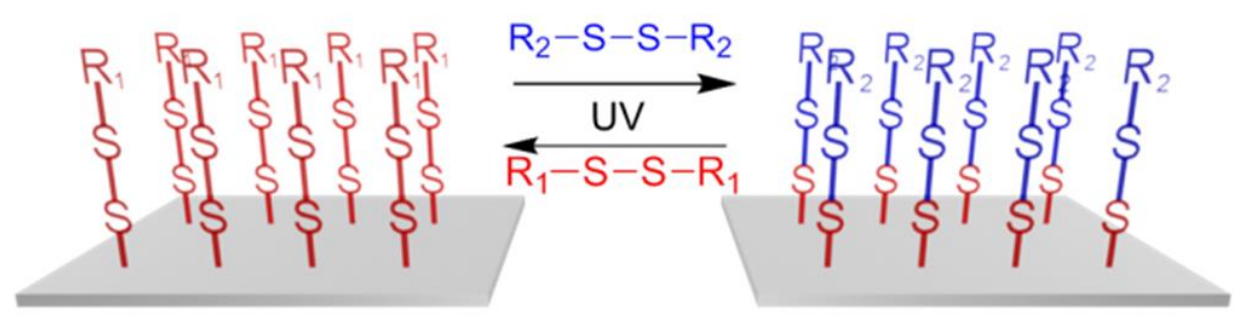

Figure 1. (a) Schematic representation of a photodynamic disulfide exchange reaction (PDDE). Disulfides are converted into two sulfanyl radicals under UV irradiation, which can combine with each other to form new disulfides. The new disulfides can again be activated to become sulfenyl radicals, and thus the process is reversible. (b) Schematic representation of the reversible surface modification based on the PDDE.

The surface disulfide exchange reaction was first characterized by WCA measurement. To enlarge the difference of WCA between different disulfide surfaces, porous substrates were used in the experiment. The disulfide surface was prepared by esterification of a porous poly(hydroxyethylmethacrylate-co-ethylene dimethacrylate) surface with bis(2-carboxyethyl) disulfide(CED) (FigureS1). The obtained CED-modified disulfide surface (CED surface) is hydrophilic with a static water contact angle (WCA) of $44 \pm 2^{\circ}$ (Figure2a). After 2 min of UV irradiation $\left(260 \mathrm{~nm}, 7.5 \mathrm{~mW} / \mathrm{cm}^{2}\right)$ in the presence of dibutyl disulfide (DBD), the static WCA increases to $128^{\circ} \pm 2^{\circ}$, indicating modification with the hydrophobic butyl sulfide groups. The produced BD surface can again be modified with CED by wetting the surface with a CED solution in DMF and irradiating with UV $\left(260 \mathrm{~nm}, 7.0 \mathrm{~mW} / \mathrm{cm}^{2}\right)$ for $2 \mathrm{~min}$, restoring the original hydrophilicity of the surface (static WCA $45^{\circ}$ ).

In order to demonstrate the reversibility of the photo-induced disulfide exchange, the reaction was repeated 20 times (10 cycles). The results shown in Figure $2 \mathrm{~b}$ confirm perfect 
reversibility of the surface modification without significant change of wettability even after 20 consecutive UV-induced functionalizations are performed on the same substrate. The slight decrease of surface WCAs of both BD and CED surfaces after 20 times of modification can be attributed to the possible surface photo-oxidation, which creates hydrophilic groups such as hydroxyl group on the surface. ${ }^{[25-27]}$ The SEM images of the disulfide surfaces reveal no morphological change during the esterification and disulfide exchange process (FigureS2).
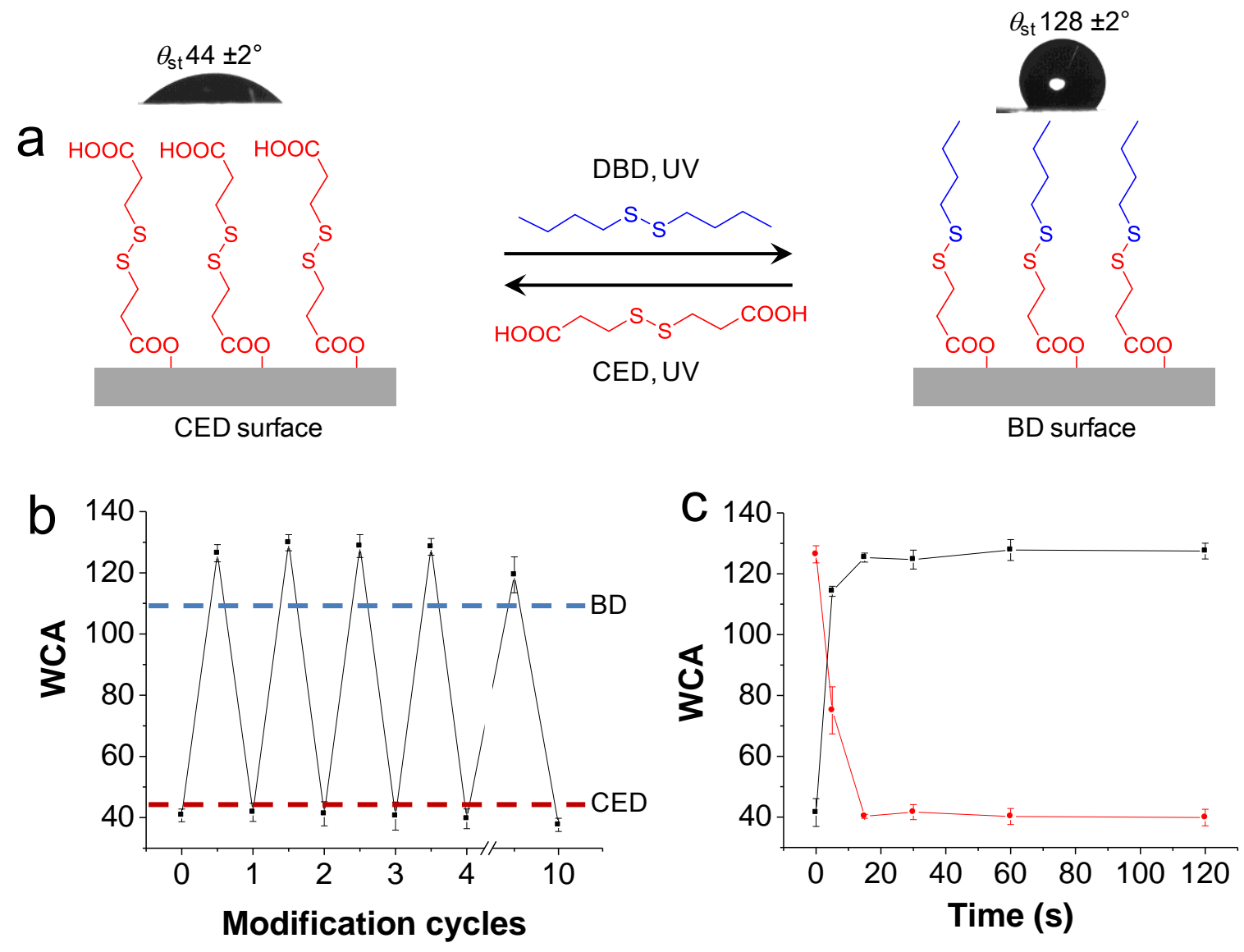

Figure 2. Kinetics and reversibility of the PDDE on a disulfide surface. (a) Schematic showing the change of surface hydrophilicity upon switching between CED and BD-surfaces using the PDDE. (b) Static WCA as a function of surface modification cycle number. The CED surface was modified with DBD under UV (1 min, $\left.7.0 \mathrm{~mW} / \mathrm{cm}^{2}, 260 \mathrm{~nm}\right)$, followed by modification of the produced BD-surface with CED. The DBD-CED modification cycle was repeated 10 times, and the WCA of the surface was measured after each modification.(c) Graph showing the static WCA as a function of irradiation time. Modification of CED surface by DBD solution. Modification of BD surface by CED solution. 
The fast kinetics of photo-induced transformations are vital for reducing irradiation time and UV damage to the surface, thereby resulting in patterns of better contrast and higher resolution. In order to elucidate the kinetics of the PDDE, a CED surface was pre-wetted with a DMF solution of DBD (20\% wt.in DMF, containing 5\% wt. DMPAP as a photoinitiator), followed by UV irradiation $\left(260 \mathrm{~nm}, 7.0 \mathrm{~mW} / \mathrm{cm}^{2}\right)$ through a quartz slide for $5 \mathrm{~s}, 15 \mathrm{~s}, 30 \mathrm{~s}$, $60 \mathrm{~s}$, and $120 \mathrm{~s}$. After irradiation was finished, the quartz glass was removed and the surface was washed with acetone, dried by $\mathrm{N}_{2}$, and static WCA was measured. Figure 2c shows that static WCAs increase from $43 \pm 4^{\circ}$ up to $114 \pm 2^{\circ}$ within the first $5 \mathrm{~s}$, followed by stabilization at $125 \pm 3^{\circ}$ at around $15 \mathrm{~s}$. The same experiment was performed using the butyl disulfide modified surface (BD surface), which was modified by the hydrophilic CED solution. The kinetics of this reaction is very similar to the modification with DBD (Figure 2c), with completeness of the reaction at $\sim 15 \mathrm{~s}$. The reaction kinetics was also investigated using different UV light intensities and wavelengths: (a) low intensity $\left(\sim 0.25 \mathrm{~mW} / \mathrm{cm}^{2}\right) 254$ and 365 $\mathrm{nm}$, and (b) medium intensity $\left(\sim 3 \mathrm{~mW} / \mathrm{cm}^{2}\right) 254$ and $365 \mathrm{~nm}$. The kinetics of the disulfide exchange was the same for both wavelengths, while the reduction of light intensity resulted in a slight decrease of the rate of transformation (Figure S3). Even in the case of a simple hand held TLC irradiation lamp and $365 \mathrm{~nm}$ UV light, surface modification was complete in 5 min.

The reversible nature of the PDDE allows for two possible surface functionalization and patterning strategies, in which a functional group can be either (a) introduced onto a disulfide surface or (b) deleted by replacing a functional group with background functionality. In order to demonstrate these possibilities, we prepared a CED disulfide surface on a HEMA-EDMA porous substrate by esterification. Figure $\mathbf{3}$ shows that the CED functionality can be conveniently replaced by a new fluorescent group (1,1'-(disulfanediylbis(ethane-2,1diyl))bis(3-(6'-hydroxy-3,3'-dioxo-3',9a'-dihydro-3H-spiro[isobenzofuran-1,9'-xanthen]-5yl)thiourea, FITC-disulfide) to generate the fluorescent FITC-disulfide surface (Figure3c). In 
the next step, the fluorescent FITC groups can be replaced with new non-fluorescent functional groups. We employed a quartz photomask to generate a micropattern of fluorescent FITC-disulfide areas (non-irradiated areas) and the newly introduced non-fluorescent butyl disulfide areas, where the surface was irradiated with UV light (Figure 3d). All of the PDDE steps were finished in less than 5 min of UV irradiation. Patterns with a variety of different shapes could easily be obtained by using different photomasks (Figure 3e). In this case, a DBsurface was utilized to directly pattern FITC-disulfide using a photomask. The smallest feature size of the produced pattern was $10 \mu \mathrm{m}$ (Figure 3f), which was limited by the porous nature of the substrate and disulfide diffusion. Increasing the viscosity of the solution and reducing the roughness of the surface can potentially increase the resolution of the patterning.

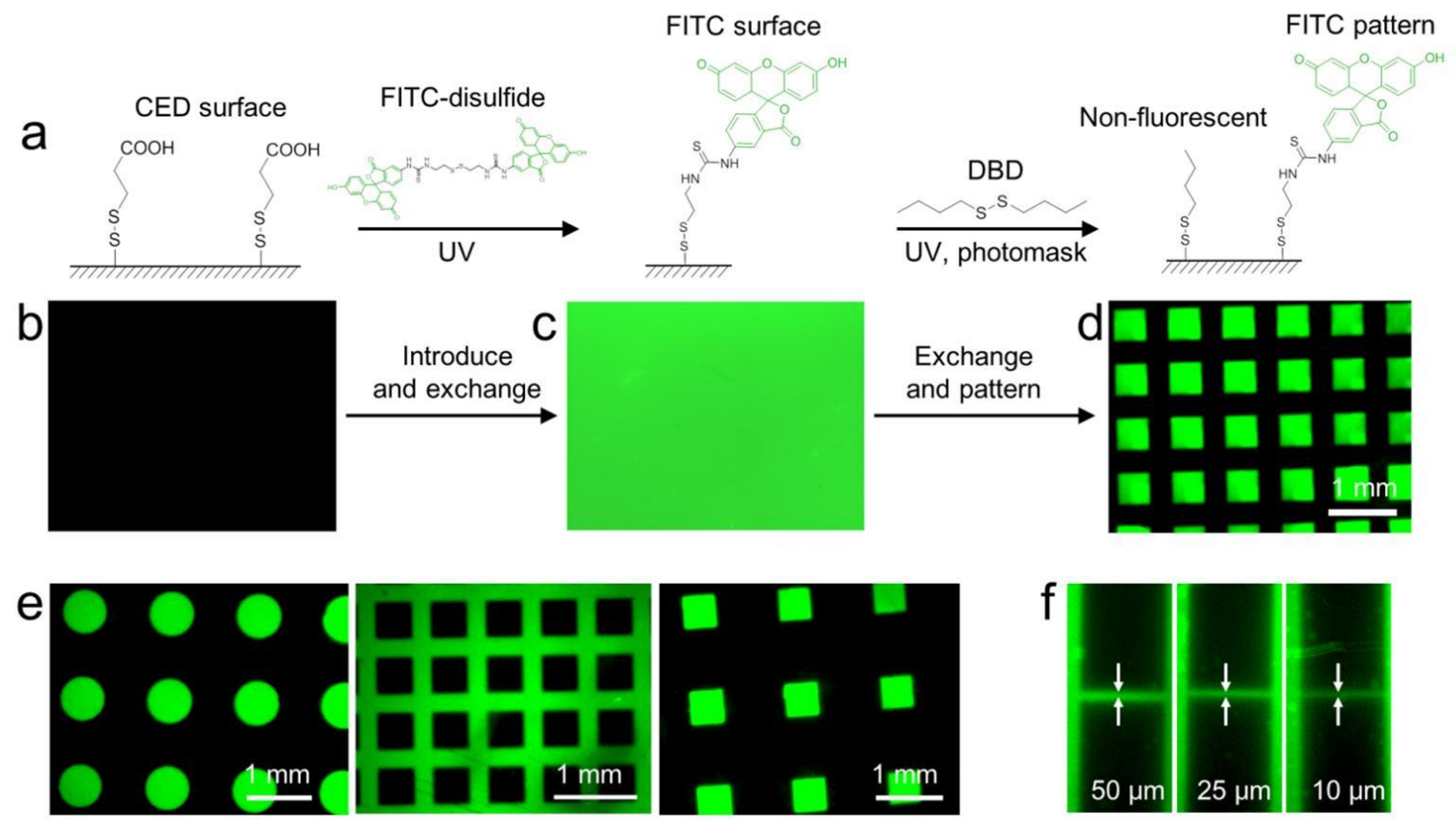

Figure 3. (a) Schematic of the surface functionalization using the PDDE. First, fluorescent FITC-labeled disulfide was introduced by replacing the CED groups with the FITC-disulfide to generate a fluorescent FITC-surface. In the second step, non-fluorescent dibutyl disulfide was patterned through a photomask on the surface by replacing the FITC-labeled disulfides.The disulfide surface was covered with a $10 \mathrm{mg} / \mathrm{mL}$ FITC-disulfide DMSO solution and irradiated with UV for $2 \mathrm{~min}$.(b, c, and d) Fluorescence microscope images of the CED, FITC-surface, and the DB-FITC patterned surfaces, respectively. (e)Patterns of FITClabeled disulfide with different geometries. FITC-disulfide was introduced by replacing the butyl disulfide modified surface (DB-surface). (f) FITC-CED patterns showing the possibility to pattern features as small as $10 \mu \mathrm{m}$ using the PDDE method. 
The PDDE was confirmed by Time-of-Flight Secondary Ion Mass Spectrometry (ToFSIMS) (Figure4). ToF-SIMS examination of a BD-CED pattern reveals a homogenous distribution of sulfur $\left(\mathrm{S}^{-}\right.$, Figure $\left.4 \mathrm{a}\right)$ and disulfide $\left(\mathrm{S}_{2}^{-}\right.$, Figure $\left.\mathrm{S} 4 \mathrm{a}\right)$ ions on the surface, while the butyl sulfide fragment is only found in the areas irradiated with UV light in the presence of DBD (Figure 4b and S4). Other corresponding ions were also detected by ToF-SIMS (Figure S4).
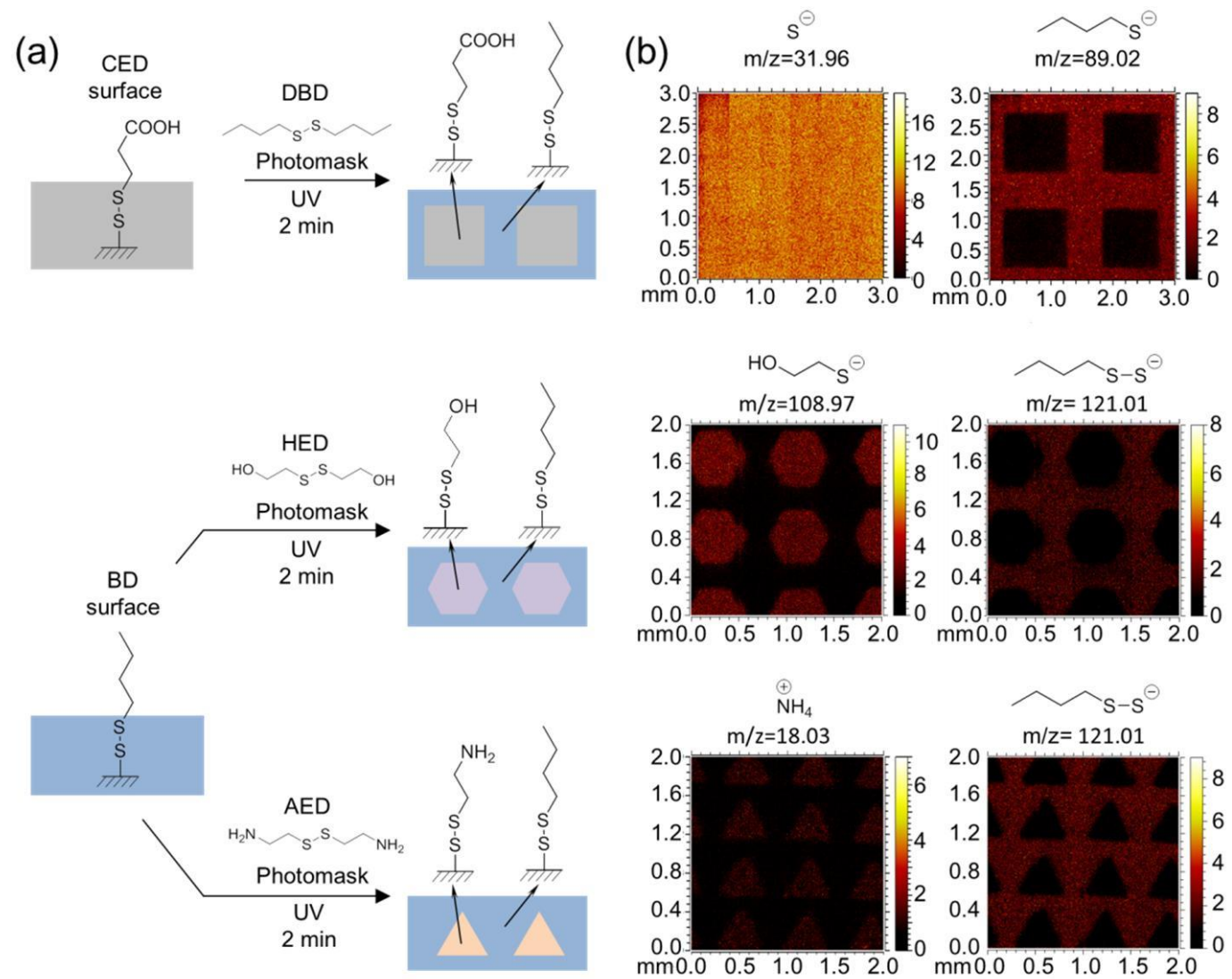

Figure 4. (a) Schematic representation of the surface patterning using the PDDE. (b) ToFSIMS maps corresponding to the surface patterns from (a).

The chemical tolerance of surface modification and patterning methods is critical for direct patterning of non-protected biomolecules and other chemicals in sensor or bioanalytical applications. The tolerance of the surface PDDE reaction to carboxy, hydroxy, and amino groups has been evaluated. ToF-SIMS results, shown in Figure 4, confirm the formation of both hydroxyl- and amino- patterns when 2-hydroxyethyl disulfide (HED) or 2-aminoethyl disulfide (AED) were used in the PDDE, respectively. 
In conclusion, we presented a new reversible surface photo-functionalization and photopatterning strategy based on a photodynamic disulfide exchange reaction. This method allows for exchange or removal of surface functional groups and can be used to create, erase or modify existing surface patterns. This approach is very versatile, fast and can be potentially exploited in various applications and development of "smart" multi-functional surfaces. We expect this strategy to be widely used in different research fields.

\section{Supporting Information}

Supporting Information is available from the Wiley Online Library or from the author.

\section{Acknowledgements}

The research is supported by the Helmholtz Association's Initiative and Networking Fund (grant VH-NG-621) and the European Research Council starting grant (ERC-2013-StG 337077-DropCellArray). X.D. and Li J. thank the China Scholarship Council for a Ph.D. scholarship.

Received: ((will be filled in by the editorial staff))

Revised: ((will be filled in by the editorial staff)) Published online: ((will be filled in by the editorial staff))

[1] A. S. Blawas, W. M. Reichert, Biomaterials 1998, 19, 595.

[2] R. Ganesan, S. Y. Yoo, J. H. Choi, S. Y. Lee, J. B. Kim, J. Mater. Chem. 2008, 18, 703.

[3] S. Morais, R. Marco-Moles, R. Puchades, An. Maquieira, Chem. Commun. 2006, 2368.

[4] Y.-K. Kim, S. R. Ryoo, S. J. Kwack, D. H. Min, Angew. Chemie Int. Ed. 2009, 48, 3507.

[5] E. J. Lee, E. W. L. Chan, M. N. Yousaf, Chembiochem 2009, 10, 1648.

[6] K. Jang, K. Sato, K. Mawatari, T. Konno, K. Ishihara, T. Kitamori, Biomaterials 2009, $30,1413$.

[7] S. J. Bryant, J. L. Cuy, K. D. Hauch, B. D. Ratner, Biomaterials 2007, 28, 2978.

[8] C. A. DeForest, B. D. Polizzotti, K. S. Anseth, Nat. Mater. 2009, 8, 659. 
[9] R. M. Hensarling, V. A. Doughty, J. W. Chan, D. L. Patton, J. Am. Chem. Soc. 2009, $131,14673$.

[10] R. T. Chen, S. Marchesan, R. A. Evans, K. E. Styan, G. K. Such, A. Postma, K. M. Mclean, B. W. Muir, F. Caruso, Biomacromolecules 2012, 13, 889.

[11] W. Song, Y. Wang, J. Qu, M. M. Madden, Q. Lin, Angew. Chemie Int. Ed. 2008, 47, 2832.

[12] T. Pauloehrl, G. Delaittre, V. Winkler, A. Welle, M. Bruns, H. G. Börner, A. M. Greiner, M. Bastmeyer, C. Barner-Kowollik, Angew. Chemie Int. Ed. 2012, 51, 1071.

[13] T. Junkers, Eur. Polym. J. 2015, 62, 273.

[14] S. V. Orski, A. A. Poloukhtine, S. Arumugam, L. Mao, V. V. Popik, J. Locklin, J. Am. Chem. Soc. 2010, 132, 11024.

[15] Y. Wang, W. Song, W. J. Hu, Q. Lin, Angew. Chemie Int. Ed. 2009, 48, 5330.

[16] H. Wu, J. Ge, M. Uttamchandani, S. Q. Yao, Chem. Commun. 2011, 47, 5664.

[17] S. Arumugam, S. V. Orski, J. Locklin, V. V. Popik, J. Am. Chem. Soc. 2012, 134, 179.

[18] S. Arumugam, V. V Popik, J. Am. Chem. Soc. 2012, 134, 8408.

[19] N. R. Gandavarapu, M. A. Azagarsamy, K. S. Anseth, Adv. Mater. 2014, 26, 2521.

[20] S. J. Rowan, S. J. Cantrill, G. R. L. Cousins, J. K. M. Sanders, J. F. Stoddart, Angew. Chemie Int. Ed. 2002, 41, 898.

[21] G. M. Whitesides, W. J. Lees, J. Org. Chem. 1993, 58, 642.

[22] P. A. Fernandes, M. J. Ramos, Chem. - A Eur. J. 2004, 10, 257.

[23] H. Otsuka, S. Nagano, Y. Kobashi, T. Maeda, A. Takahara, Chem. Commun. 2010, 46, 1150 .

[24] Y. Amamoto, H. Otsuka, A. Takahara, K. Matyjaszewski, Adv. Mater. 2012, 24, 3975.

[25] C. M. Chan, T. M. Ko, H. Hiraoka, Surf. Sci. Rep. 1996, 24, 1.

[26] I. Mathieson, R. H. Bradley, Int. J. Adhes. Adhes. 1996, 16, 29.

[27] D. Zhang, S. M. Dougal, M. S. Yeganeh, Langmuir 2000, 16, 4528. 
Table of contents: Reversible surface photo-functionalization and photo-patterning strategy based on a photodynamic disulfide exchange reaction is demonstrated. The method allows for rapid and reversible functionalization, patterning, as well as exchange or removal of functional groups on the surface.

Keywords: patterning; surface functionalization; photochemistry; disulfide exchange; rewritable surface

Xin Du, Linxian Li, Junsheng Li, Alexander Welle, and Pavel A. Levkin*

\section{Reversible and Rewritable Surface Functionalization and Patterning via Photodynamic Disulfide Exchange}

ToC figure

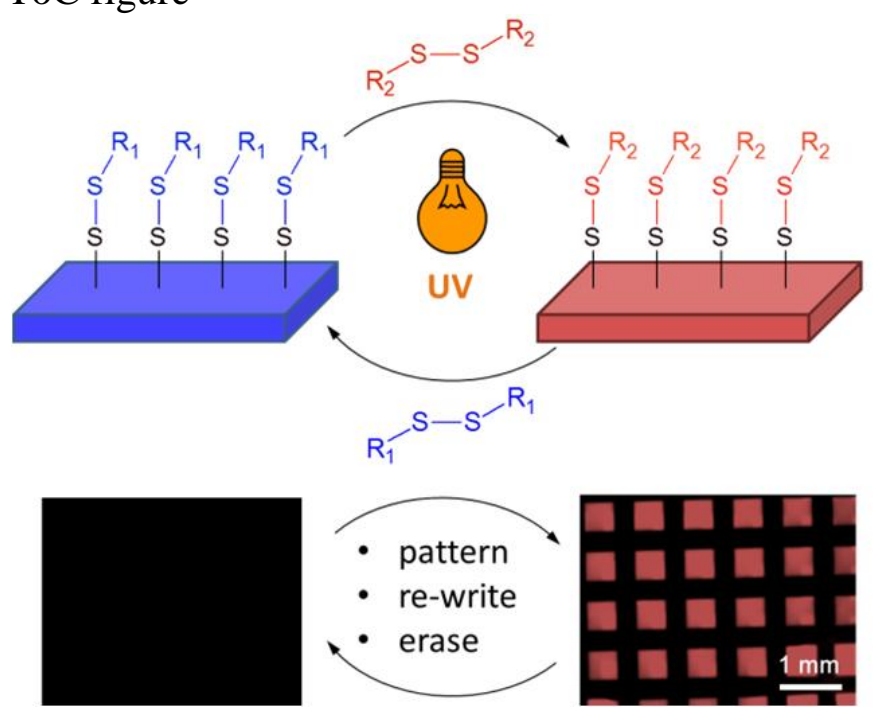


Copyright WILEY-VCH Verlag GmbH \& Co. KGaA, 69469 Weinheim, Germany, 2013.

\section{Supporting Information}

\section{Reversible and Rewritable Surface Functionalization and Patterning via Photodynamic Disulfide Exchange}

Xin Du, Linxian Li, Junsheng Li, Alexander Welle, and Pavel A. Levkin*

\section{Experiment}

\section{Materials}

Dibutyl disulfide (DBD), 2-hydroxyethyl disulfide (HED), 2-Carboxyethyl disulfide (CED), 2,2-Dimethoxy-2-phenylacetophenone (DMPAP), fluorescein isothiocyanate isomer I (FITC), N,N'-Diisopropylcarbodiimide (DIC), cystamine dihydrochloride, 2-hydroxyethyl methacrylate, and ethylene glycol dimethacrylate were obtained from Sigma-Aldrich (Germany). 4-(Dimethylamino)pyridine (DMAP), ethanol, dichloromethane (DCM), dimethyl sulfoxide (DMSO), acetone, dimethylformamide (DMF), and other solvents were obtained from Merck KGaA (Germany). Didodecyl disulfide (DoD) was synthesized according to the literature. ${ }^{[1]}$ The glass plates used in the experiment were Nexterion B glass from Schott AG (Germany). An OAI model 30 deep-UV collimated light source (San Jose, CA, USA) fitted with a $500 \mathrm{~W} \mathrm{HgXe}$ lamp was utilized for high intensity UV irradiation. The lamp was calibrated to $7.0 \mathrm{~mW} \mathrm{~cm}^{-2}$ at $260 \mathrm{~nm}$ with the OAI $306 \mathrm{UV}$ power meter. For medium intensity UV irradiation, a BIO-LINK BLX UV chamber (LaborTechnik GmbH \& Co., KG, Germany) equipped with a 5 x 8W UV lamp (wavelength: 254 and $365 \mathrm{~nm}$, respectively) was used as the light source. For weak intensity UV irradiation, a UVGL-25 compact UV lamp (254/365 nm, 4W, USA) was used as the light source. The microscopy images were obtained by a BIOREVO BZ-9000 microscope (Keyence GmbH, Germany).

FITC-disulfide was synthesized as follows: Cystamine dihydrochloride $(4 \mathrm{mg})$ and triethylamine $(8 \mu \mathrm{L})$ were mixed in $2 \mathrm{ml} \mathrm{DCM}$, and vortexed until cystamine dihydrochloride 
was dissolved. The cystamine solution was added into a FITC solution (13 mg dissolved in 1 $\mathrm{mL}$ of DMSO). After the addition of $12 \mathrm{mg}$ of DMAP, the mixture was stirred for $16 \mathrm{~h}$ to obtain FITC-disulfide. The FITC-disulfide solution was evaporated by Biotage V10 to remove DCM, and diluted with DMSO to a concentration of $10 \mathrm{mg} / \mathrm{mL}$.

\section{Formation of the disulfide surface}

The carboxyethyl-disulfide surface (CED surface) was formed by esterification of CED on the HEMA-EDMA surface (Figure S1, the formation of micro- and nano-porous HEMAEMDA could be found in our previous report). ${ }^{[2]}$ First, $240 \mathrm{mg}$ of CED were added into a 50 $\mathrm{ml}$ Falcon tube containing $45 \mathrm{ml}$ of acetone, followed by the addition of $176.5 \mu \mathrm{L}$ of DIC. Then, the porous HEMA-EDMA surface was placed into the tube, and the solution was stirred with a small magnetic stirrer for $7 \mathrm{~h}$ after the addition of $56 \mathrm{mg}$ DMAP. The HEMA-EDMA surface was finally washed with ethanol and acetone, and dried by $\mathrm{N}_{2}$.

Butyl disulfide surface (BD surface) was formed by UV exchange of DBD with CED surface. To achieve this, a few drops of DBD solution (20\% wt in DMF, containing $1 \%$ wt DMPAP as a photoinitiator) were added onto the CED surface, and then the surface was covered by quartz glass and irradiated under UV for 2 min. The surface was obtained after washing with acetone and drying with $\mathrm{N}_{2}$. Hydroxyethyl disulfide surface (HED surface) was formed by the same procedure with HED as the exchange disulfide.

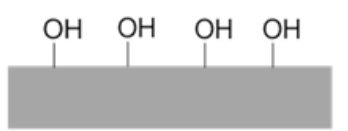

$$
+\mathrm{HOOC}_{\mathrm{S}} \sim \mathrm{COOH} \frac{\mathrm{DIC}, \mathrm{DMAP}(1 \mathrm{eq})}{\text { Acetone, } 7 \mathrm{~h}}
$$

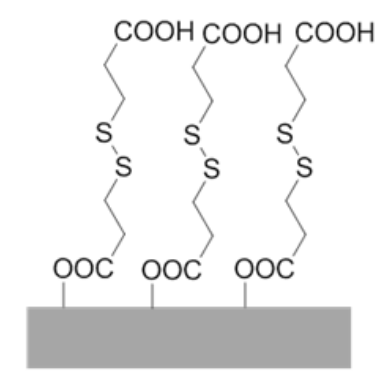

HEMA-EDMA

Figure S1. Preparationof the disulfide surface. 


\section{SEM test on the disulfide surface}

SEM images were obtained using the LEO 1530 Gemini scanning electron microscope (Zeiss, Germany) at the Institute of Nanotechnology (INT), KIT. The samples were sputtered with a $30 \mathrm{~nm}$ gold layer using a Cressington 108 auto sputter coater (INT, KIT) before the measurement.

\section{Photo-patterning on the disulfide surface}

A few drops of disulfide solution (20\% wt. in DMF with $1 \%$ wt. DMPAP, forFITCdisulfide is $10 \mathrm{mg} / \mathrm{mL}$ in DMSO containing $0.5 \mathrm{mg} / \mathrm{mL}$ DMPAP) were added to the disulfide surface, and then the surface was covered by quartz glass and irradiated under UV for 2 min with a photomask. The surface was then washed with acetone and dried by $\mathrm{N}_{2}$.

For the reverse patterning of FITC-disulfide, the disulfide surface was first flood irradiated by UV for 5 min, followed by a wash process. Then, a few drops of DBD solution were added on the surface, and the surface was irradiated under UV for 2 min with a photomask. Afterwards, the surface was washed in acetone overnight and dried by $\mathrm{N}_{2}$.

\section{Time-of-Flight Secondary Ion Mass Spectrometry (ToF-SIMS)}

Images were obtained with the TOF-SIMS 5 machine (ION-TOF GmbH, Münster, Germany). The analysis chamber was held at $\sim 8 \times 10^{-9}$ mbar during the experiment. A pulsed $25 \mathrm{keV} \mathrm{Bi}_{1}{ }^{+}$primary ion beam was used for all image and spectral data acquisition. All data were collected in high mass resolution bunched mode. Care was taken to use a new sample area for each analysis. 


\section{SupplementaryFigures}

a
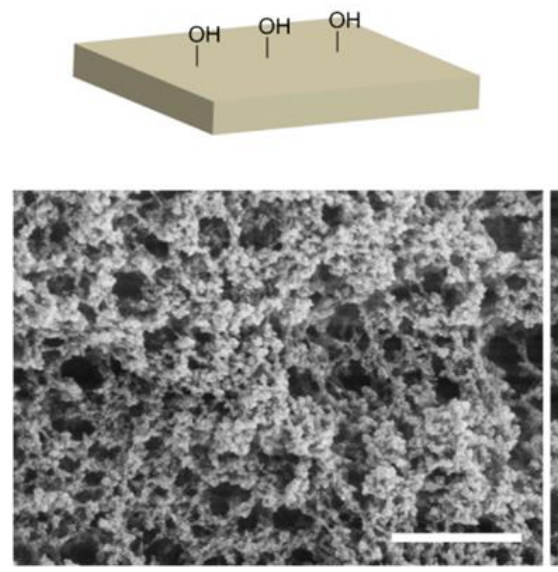

b
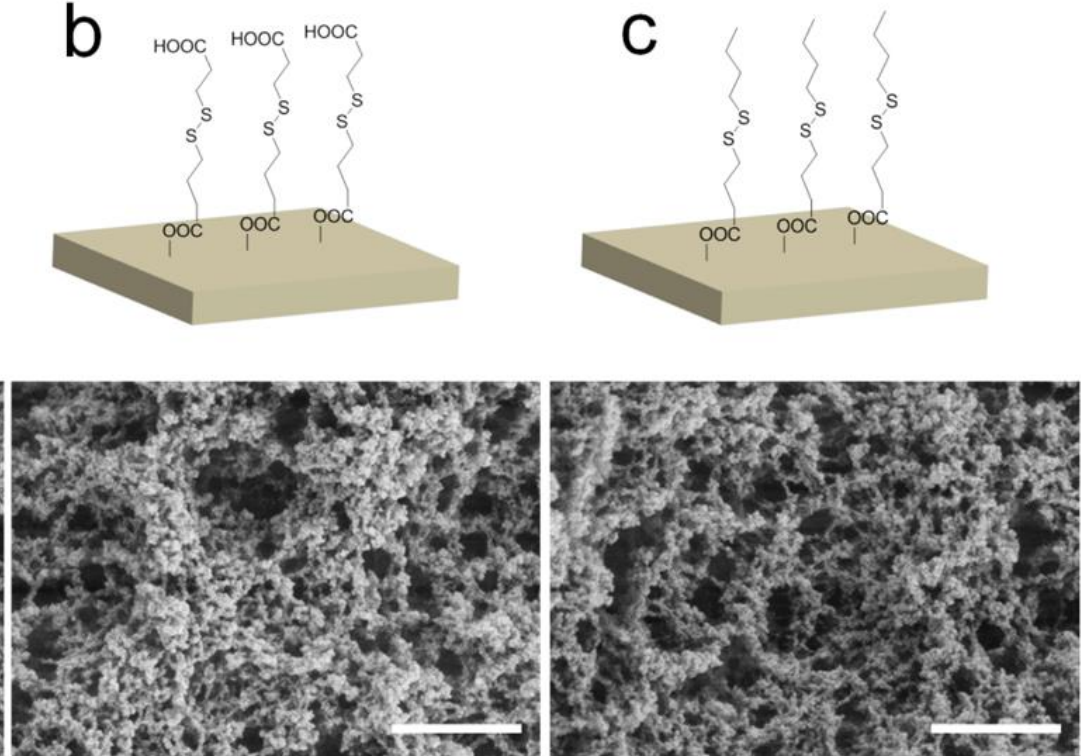

Figure S2. SEM images of the HEMA-EDMA surface before and after disulfide modification.

No differences could be found regarding the morphology of the surface. (a) HEMA-EMDA surface. (b) CED surface after the esterification of HEMA-EDMA with CED. (c) BD surface after disulfide exchange on CED surface. The scale bars in the images refer to $1 \mu \mathrm{m}$. 

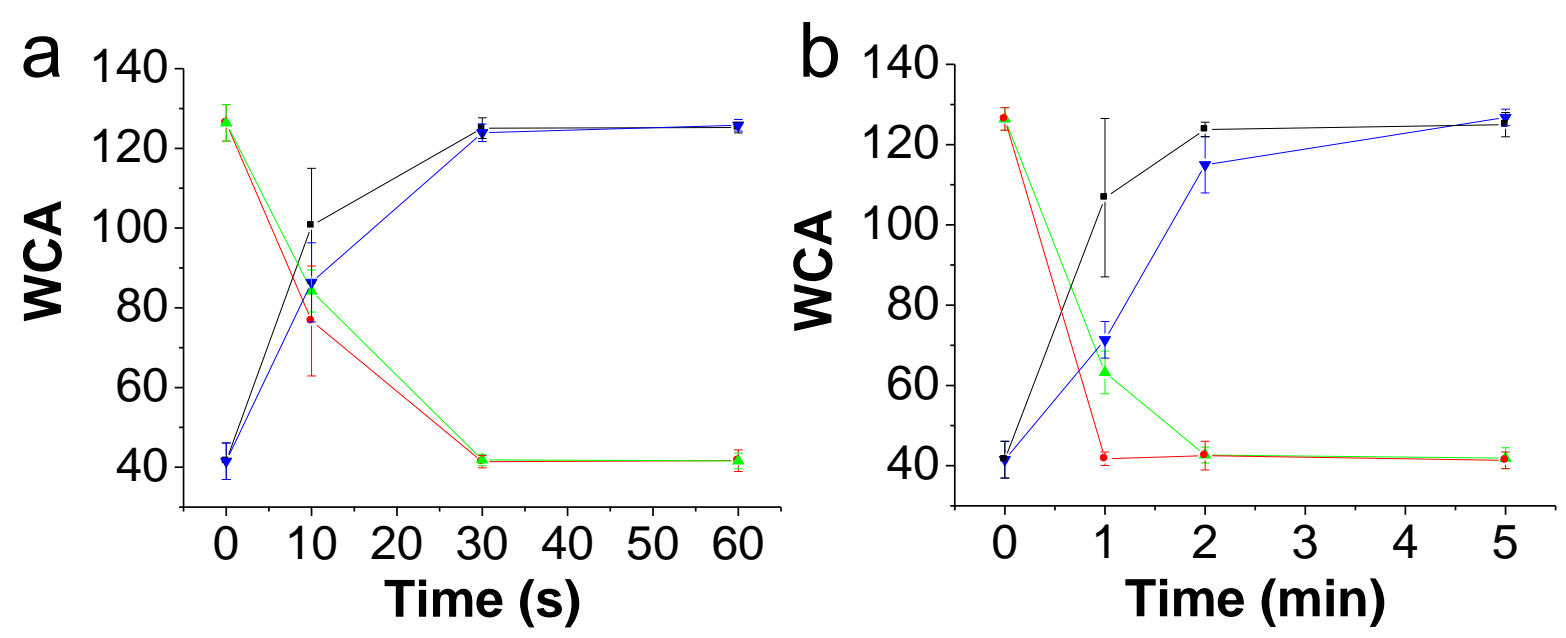

Figure S3. Kinetics of photodynamic disulfide exchange on the surface. (a) Disulfide exchange under medium $\left(\sim 2 \mathrm{~mW} / \mathrm{cm}^{2}\right)$ UV intensity at the wavelength of $254 \mathrm{~nm}$ and $365 \mathrm{~nm}$, respectively. Samples: $\mathbf{C E D}$ surface modified by DBD solution under $254 \mathrm{~nm}$ UV light. BD surface modified by CED solution under $254 \mathrm{~nm}$ UV light. $\nabla$ CED surface modified by DBD solution under $365 \mathrm{~nm}$ UV light. $\triangle$ BD surface modified by CED solution under 365 nm UV light. (b) Disulfide exchange under weak $\left(\sim 0.2 \mathrm{~mW} / \mathrm{cm}^{2}\right)$ UV intensity at the wavelength of $254 \mathrm{~nm}$ and $365 \mathrm{~nm}$, respectively. CED surface modified by DBD solution under $254 \mathrm{~nm}$ UV light. $\bullet$ BD surface modified by CED solution under $254 \mathrm{~nm}$ UV light. CED surface modified by DBD solution under $365 \mathrm{~nm}$ UV light. $\triangle$ BD surface modified by CED solution under $365 \mathrm{~nm}$ UV light. 


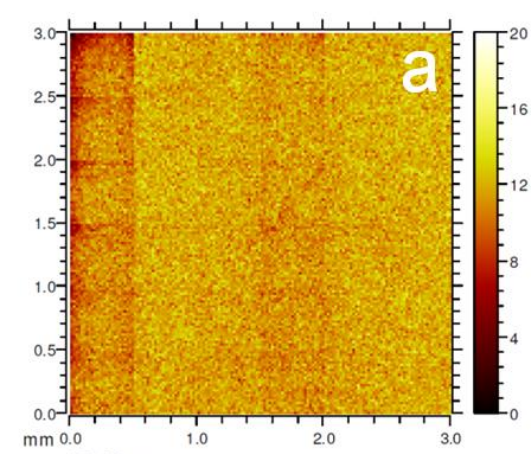

$63.93 \mathrm{u}$

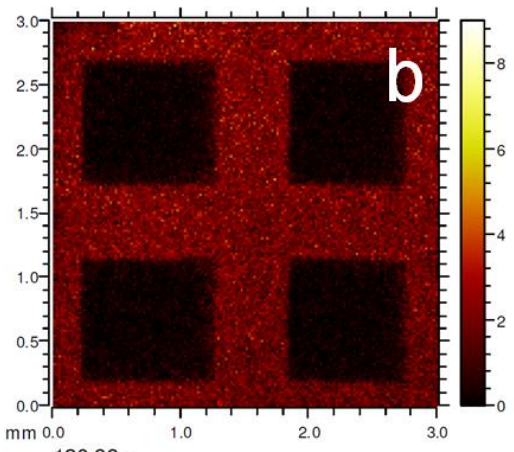

$120.98 \mathrm{u}$

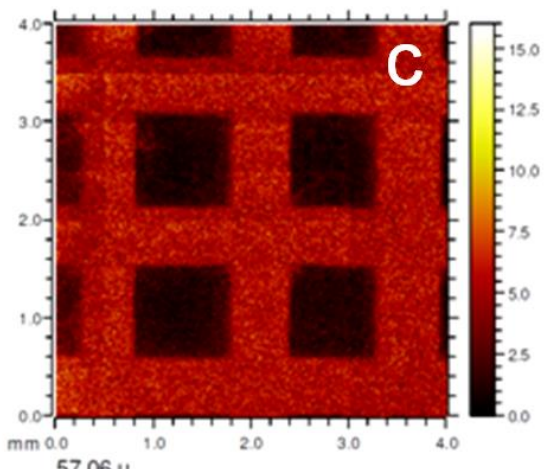

57.064

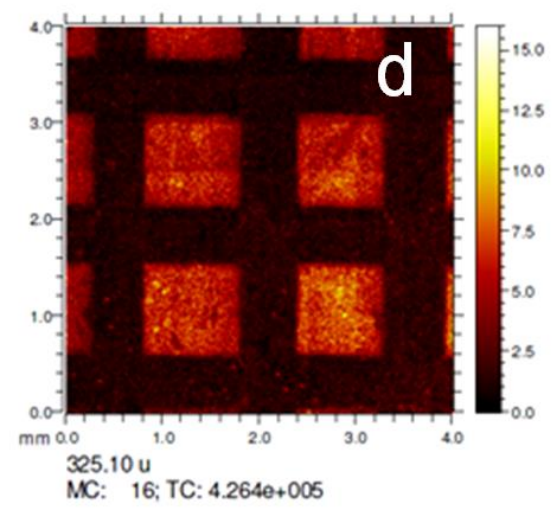

$\stackrel{\ominus}{\mathrm{S}_{2}}$

Chemical Formula: $\mathrm{S}_{2}{ }^{-}$

Molecular Weight: 64.12

m/z: 63.94 (100.0\%), 65.94 (9.0\%), 64.94 (1.6\%)<smiles>CCCCS[S-]</smiles>

Chemical Formula: $\mathrm{C}_{4} \mathrm{H}_{9} \mathrm{~S}_{2}{ }^{-}$

Exact Mass: 121.02

m/z: 121.02 (100.0\%), 123.01

(4.5\%), $123.01(4.5 \%), 122.02$

(4.3\%), $122.01(1.6 \%)$<smiles>[C+]CCC</smiles>

Chemical Formula: C4H9+

Molecular Weight: 57.12 m/z: 57.07 (100.0\%), 58.07 (4.3\%)<smiles>CC(C)C(=[OH+])OCCOC(=O)CCSSCCC(=O)O</smiles>

Chemical Formula: C12H21O6S2+

Molecular Weight: 325.41 $\mathrm{m} / \mathrm{z}: 325.08$ (100.0\%), 326.08 (13.0\%), 327.07 (9.0\%), 326.08 (1.6\%), 327.08 (1.2\%), $328.08(1.2 \%)$

Figure S4. ToF-SIMS mapping results on a patterned BD-CED surface. The pattern was formed by UV irradiation on CED surface in DBD solution with a photomask. (a) and (b) are in negative polarity, and (c) (d) are in positive polarity. The negative and positive imaging runs were performed in different areas. (a) Mapping result of the fragment with $\mathrm{m} / \mathrm{z}=63.93$, 
which refers to disulfur anion. No pattern could be observed since the sulfur is homogeneously distributed on the surface. (b) Mapping result of the fragment with $\mathrm{m} / \mathrm{z}=120.98$, which refers to the fragment of butyl disulfide anion. A clear pattern can be observed on the irradiated area. (c) Mapping result of the fragment with $\mathrm{m} / \mathrm{z}=57.06$, which refers to butyl cation from butyl sulfide chain. Only the irradiated area showed a signal of this fragment. (d) Mapping result of the fragment with $\mathrm{m} / \mathrm{z}=325.1$, which probably refers to a CED-HEMA side chain derivative. Only the non-irradiated area showed a signal of this peak.

\section{References}

[1] M. Kirihara, Y. Asai, S. Ogawa, T. Noguchi, A. Hatano, Y. Hirai, Synthesis, 2007, 21, 3286.

[2] P. A. Levkin, F. Svec, J. M. J. Frechet, Adv. Funct. Mater.2009, 19, 1993. 